\title{
Retreatment of Silver Point Obturation: A Case Report and an Overview
}

Shikha Gulati, MV Sumanthini, Vanitha Shenoy

\begin{abstract}
Clinicians frequently encounter endodontically treated teeth that contain silver points within their roots. If endodontic treatment is failing, the need arises to remove this metallic obstruction to facilitate successful nonsurgical retreatment. For the retrieval of these obstructions, most of the methods suggest creating and enlarging a space around the obstruction to loosen it before its retrieval. A large number of removal techniques exists including use of a variety of appropriate burs, specialized forceps, ultrasonic instruments in direct or indirect contact, peripheral filing techniques in the presence of solvents, chelators, or irrigants and microtube delivery using mechanical, adhesion techniques. This case report involves the use of several Hedstrom files, inserted and twisted along the length of silver point to entangle the point and withdraw it, with an overview of other techniques of removal of silver point.
\end{abstract}

Keywords: Retreatment, Canal obstruction, Retrieval, Silver point removal, Bleaching.

How to cite this article: Gulati S, Sumanthini MV, Shenoy V. Retreatment of Silver Point Obturation: A Case Report and an Overview. J Contemp Dent 2012;2(3):114-118.

\section{Source of support: Nil}

Conflict of interest: None declared

\section{INTRODUCTION}

Endodontic obturation with silver points was introduced during the 1930's. Jasper in year 1933 introduced silver point having same diameter as files and reamers. ${ }^{1}$ Historically, the use of silver point was popular because of their ease of handling and placement, ductility, radiopacity and some antibacterial property. However, over the past few decades, the use of silver point has dramatically diminished. Silver points do not produce an acceptable three dimensional seal of the root canal system; rather they simply produce a plug in the apical constriction, with poor adaptability to the root canal walls and do not seal the accessory canals that are frequently present. Also, silver points corrode over time which again compromises the apical seal. ${ }^{2}$ The fact remains that inspite of the disadvantages of silver points they are still in use and clinicians frequently encounter endodontically treated and failed teeth that contain silver points within their roots.

In failing root canal cases, it is of foremost importance to differentiate silver point obturation from gutta percha obturation radiographically, wherein, silver point appears parallel over its length and more radiopaque than gutta percha. ${ }^{1}$ When the elected treatment plan is endodontic nonsurgical retreatment, then the goal is to access the pulp chamber, remove material from root canal system, reshape and clean the canal and obturate with gutta percha.

There has been rapid advancement in endodontic retreatment in recent years. Currently, there are various techniques available to remove silver point or any other metallic obstructions, which are encountered in the root canal. It includes use of a variety of appropriate burs, specialized forceps, ultrasonic instruments in direct or indirect technique, peripheral filing technique in presence of solvents, chelators, or irrigants and microtube delivery using mechanical adhesion techniques. ${ }^{3}$

The case report describes the nonsurgical retreatment of a maxillary central incisor obturated with silver point and illustrates a few silver point removal techniques.

\section{CASE REPORT}

A 24-year-old female patient reported to the Department of Conservative Dentistry and Endodontics with a chief complaint of mild pain and discoloration in maxillary front tooth since 1 month and gave history of root canal treatment done 1 year ago. Clinical examination revealed that the right maxillary central incisor (tooth no 11) was tender on percussion, slightly discolored, periodontal probing within normal limits $(<3 \mathrm{~mm})$ and no mobility. Fractured and discolored composite resin restorations were observed on the mesial surfaces of 11 and 21 (maxillary left central incisor), as shown in Figure 1. Preoperative radiograph revealed silver point obturation and thickening of periodontal ligament space in 11 (Fig. 2).

Based on clinical and radiographic examination, a diagnosis of chronic apical periodontitis was made. Patient's

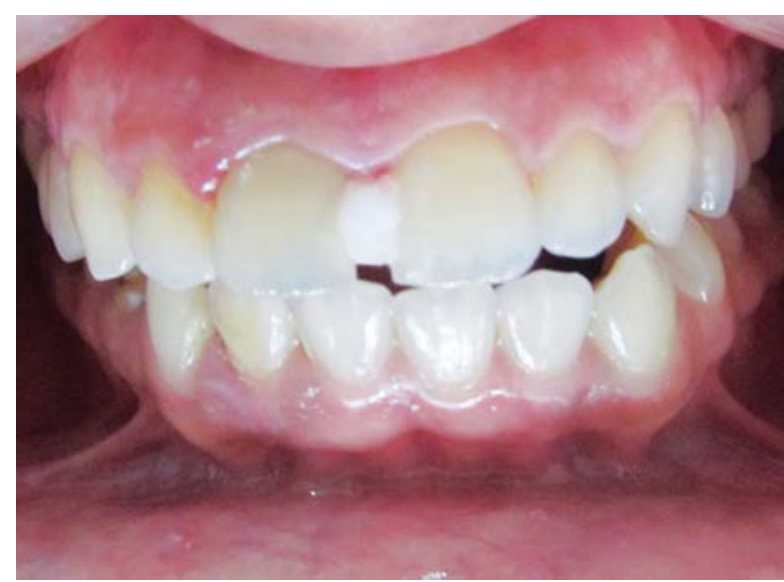

Fig. 1: Preoperative photograph showing faulty composite resin restoration on mesial surface of 11 and 21 and discolored 11 
medical history was noncontributory. Nonsurgical endodontic retreatment followed by walking bleach was recommended. During all steps of treatment, tooth was isolated with rubber dam.

Under rubber dam isolation coronal restoration was removed with high speed handpiece taking care not to damage the coronal aspect of silver point. Once proper access was established, it was flooded with gutta percha solvent (Xylene, Dentsply Maillefer DMS IV, Dentsply Maillefer Ballaigues, Switzerland) to soften or dissolve the sealer, which enabled easier removal of silver point. A no 10 stainless steel K-file (MANI, INC. Japan) was used to carry solvent down along the length of the silver point to dissolve as much sealer as possible. Fresh solvent was then introduced. It was repeatedly done, since fresh solvent enhances the efficiency of sealer removal. After thorough removal of sealer, three \#10 Hedstrom files (MANI INC. Japan) were inserted down along the silver point as apically as possible in mesial, distal and labial areas. The files were then twisted around one another to entangle the silver point and withdrawn as one unit (Figs 3 and 4). Confirmatory radiograph was taken to check the complete removal of silver point (Fig. 5).

The root canal was then cleaned and shaped with $2 \% \mathrm{~K}$ and $\mathrm{H}$ hand files with step back technique to master apical file (MAF) no $45 \mathrm{~K}$-file. Canal was intermittently irrigated with $2.5 \%$ sodium hypochlorite (Prime Dental Products, Thane, India). Calcium hydroxide (Deepashree Products, Ratnagiri, India) and chlorhexidine (Vishal, V-concept Dentocare Pvt Ltd, Ahmedabad, Gujarat, India) were mixed to obtain workable consistency and densely packed inside the root canal. Tooth was then temporized with zinc oxide eugenol cement (Deepak Enterprise, Mumbai, India). At the 1 week recall, patient was asymptomatic and tooth was obturated with gutta-percha (Dentsply Maillefer, Ballaigues, Switzerland) and AH plus sealer (Dentsply Maillefer Dentsply, Konstanz, Germany) by lateral condensation

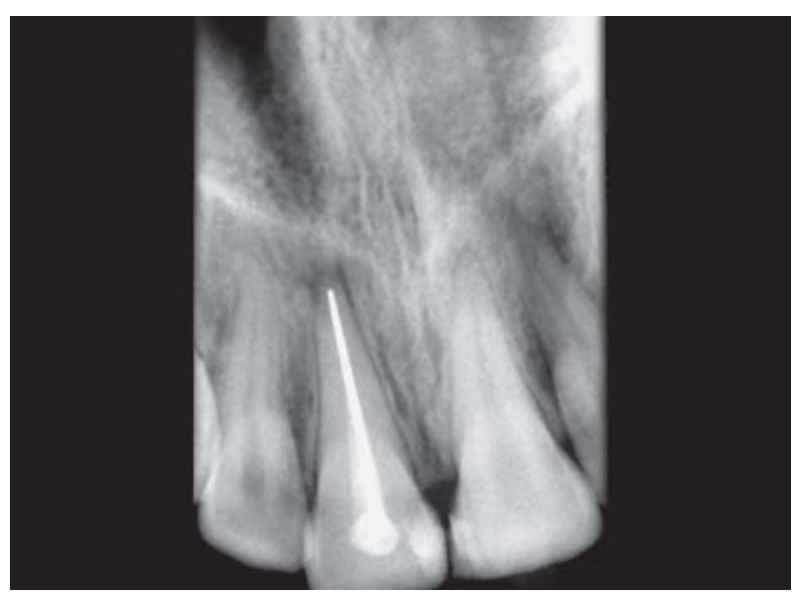

Fig. 2: Preoperative radiograph showing silver point obturation and thickening of periodontal ligament space in 11

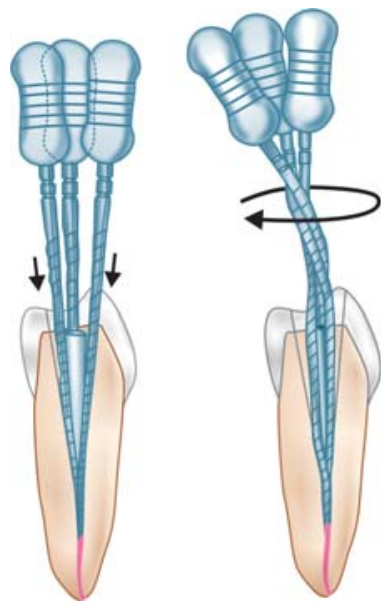

Fig. 3: Braiding technique

(Courtesy: Ingle JI, Bakland LK, Endodontics (5th ed). Hamilton, London: BC Decker Inc 2002;648)

technique followed by temporization with zinc oxide eugenol cement (Fig. 6).

In the subsequent visit, nonvital tooth bleaching was initiated to treat the discoloration. Preoperative color of the tooth was recorded (A3) using shade guide (VITA, Bad Sackingen, Germany), as it provides a point of reference for future comparison. Gutta-percha was removed $2 \mathrm{~mm}$ below the cementoenamel junction and a barrier of zinc polycarboxylate cement (Ploy- F, Dentsply Maillefer, Ballaigues, Switzerland) was placed (Figs 7A and B). Sodium perborate was mixed with $30 \%$ hydrogen peroxide (Merck Specialities Pvt Ltd, Mumbai, India) in a clean dappen dish to obtain a thick past. The paste was carried into the pulp chamber with a plastic filling instrument taking care to cover the entire facial surface of the pulp chamber with the paste. Tooth was then sealed with interim restorative material (Dentsply, Milford, USA). The procedure was repeated twice with a gap of 1 week. It was observed that the color of the tooth changed from shade A3 to A2. It was decided to stop the bleaching procedure as tooth color was similar to the adjacent teeth. Residual peroxides from

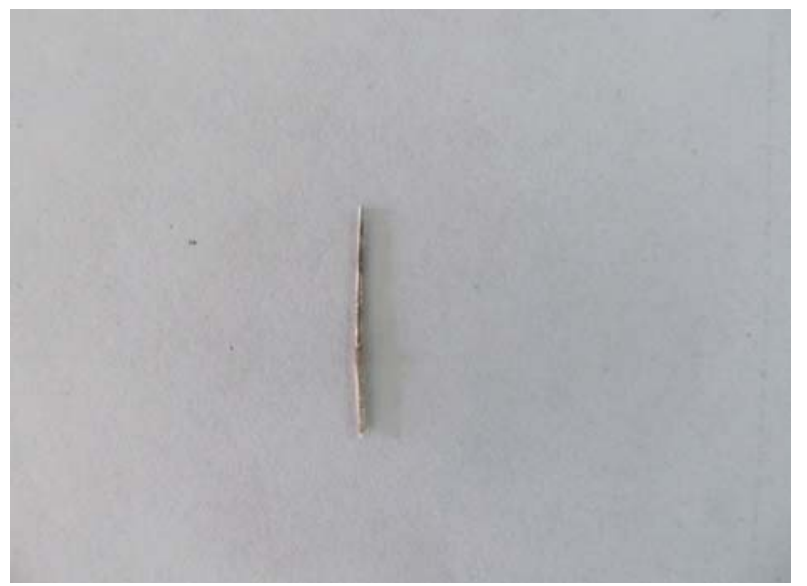

Fig. 4: Silver point removed from the root canal 
bleaching agent (mainly hydrogen peroxide) can affect the bonding strength of composite resins. Therefore, tooth was sealed with interim restorative material and patient was recalled after 3 weeks. During the following visit, fractured composite resin restoration on the mesial of 21 , and access cavity space and mesial of 11 were restored with composite resin (Dentsply Ceram x Duo, Konstanz, Germany) as

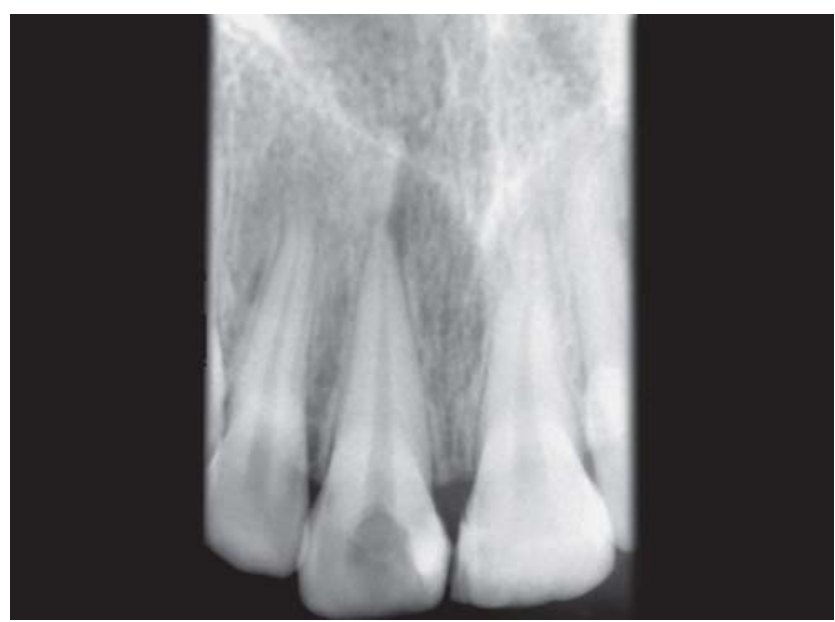

Fig. 5: IOPA radiograph confirming removal of silver point

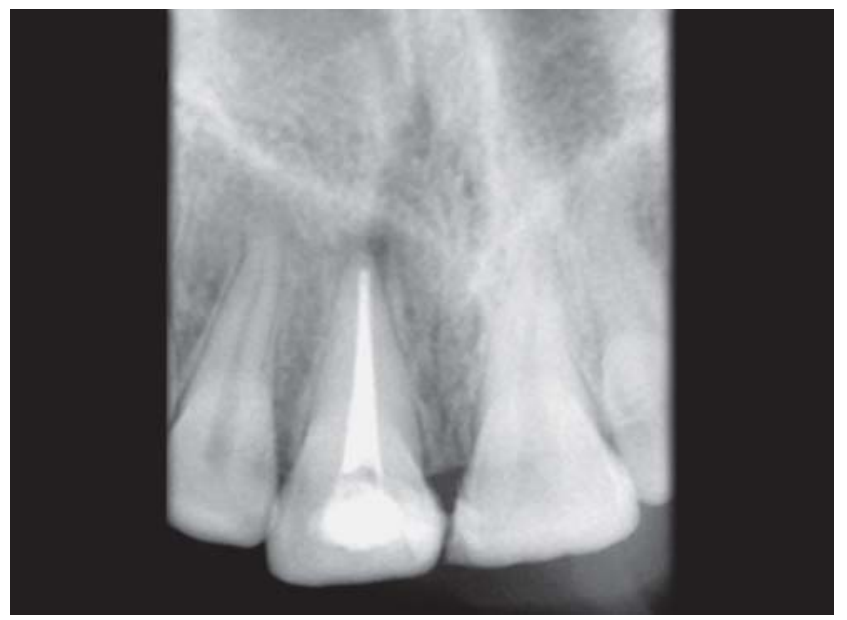

Fig. 6: Eleven obturated with gutta-percha and AH Plus sealer

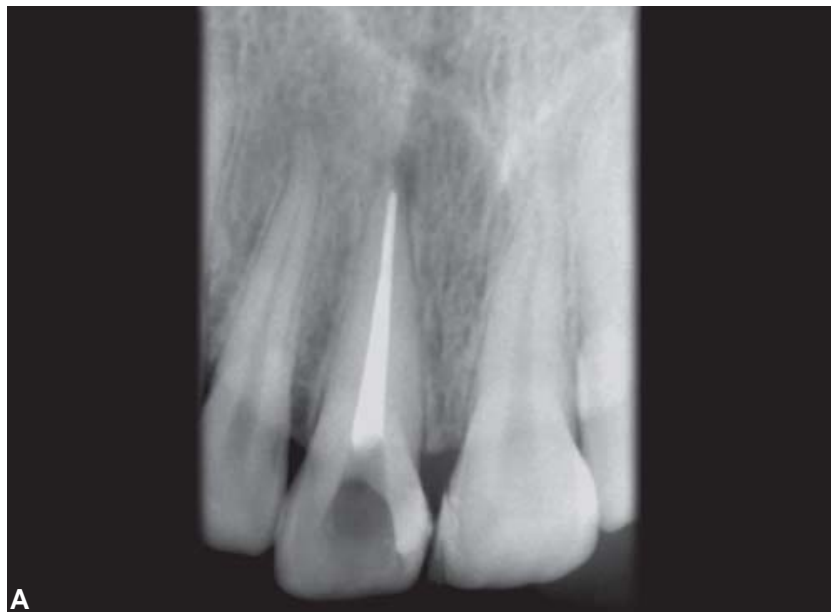

shown in Figure 8. Radiograph was taken to confirm the density of composite resin (Fig. 9). At the 3-month followup, patient was asymptomatic and periapical radiograph showed signs of healing (Fig. 10).

\section{DISCUSSION}

Silver points lack plasticity to flow and confirm to the shape of the root canal system. It is one of the reasons for diminished use of silver points from last few decades. There are some additional clinically practical problems with the use of silver point: (i) Post and core build up becomes impossible with intact silver point, necessitating retreatment and replacement of the points with another material. (ii) Apical surgery becomes more complicated due to differences encountered when attempting a root end preparation in canals that are filled with metal. (iii) Corrosion products, which cause argyrosis and periradicular inflammation, have the potential to induce inflammatory root resorption. ${ }^{4}$ Silver points corrode in the presence of tissue fluids and certain chemicals used during endodontic treatment, which includes sodium hypochlorite and some sealers. The corrosion byproducts such as silver sulfide, silver sulfate, silver carbonate, silver amine hydrate can cause staining of the tooth structure. ${ }^{2}$ Though literature supports the decreased usage of silver point, yet it is in clinical usage because of its ease of handling and placement, ductility, radiopacity and some antibacterial activity. With the introduction of these rigid silver cones it became possible to easily place them to length even in curved, narrow canals with little preparation. This resulted in clinicians often failing to properly clean and shape the canal before obturation. Therefore, treatment failures were mainly the result of the leakage and the failure to remove the irritants from the root canal system. ${ }^{2}$

Silver point canal preparation techniques produce a relatively parallel preparation in the apical 2 to $3 \mathrm{~mm}$ of the

Figs 7A and B: $2 \mathrm{~mm}$ of coronal GP was removed and space restored with zinc polycarboxylate cement 
canal, and flared coronal to this apical zone. When clinicians evaluate silver point failures and subsequent retreatment strategies, they should recognize that the silver point have minimal taper and are smooth sided. In a coronally shaped canal, one may take advantage of this available space when approaching retreatment. ${ }^{1}$

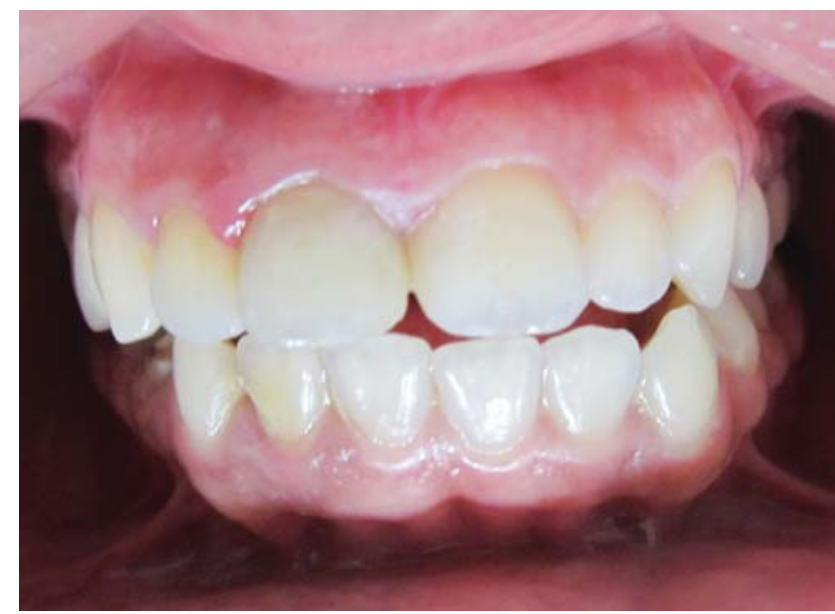

Fig. 8: Postoperative photograph showing effect of bleaching on 11 and composite resin build up on mesial surface of 11 and 21

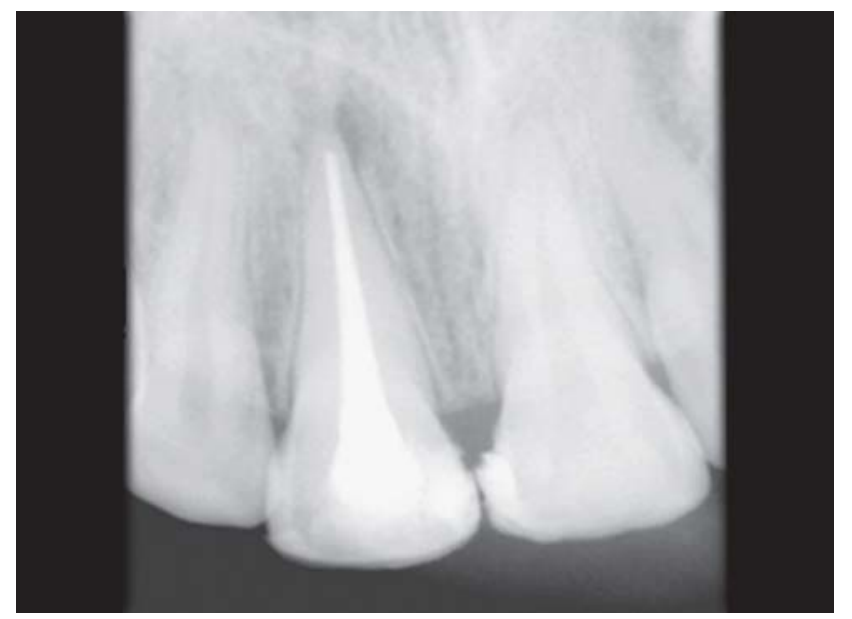

Fig. 9: Postoperative radiograph

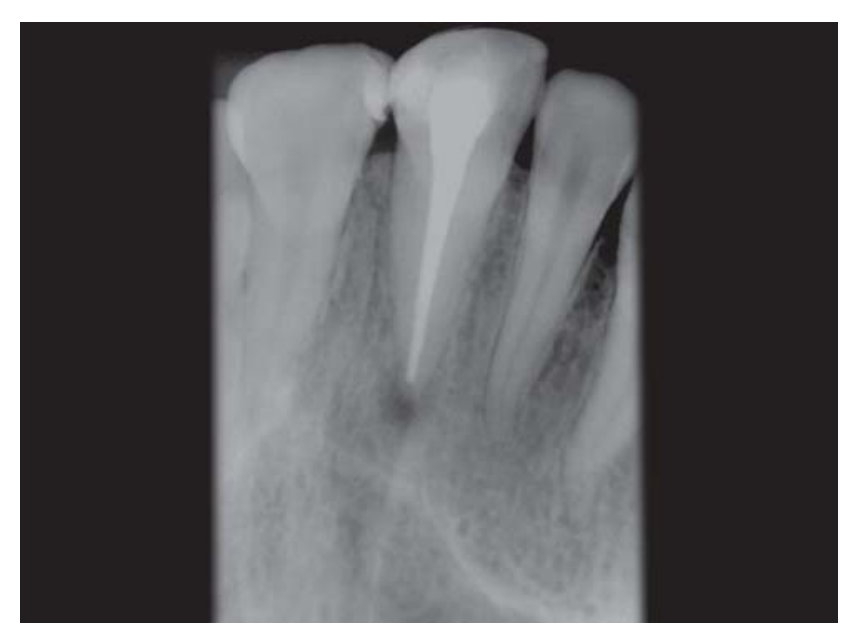

Fig. 10: Three-month follow-up radiograph showing signs of healing
During retreatment of silver point obturated root canals, immediate removal of the silver points should never be attempted. Even if a silver point appears to be poorly adapted to the coronal two- thirds of the canal, it may fit well in the apical third. Silver points will often be affected by corrosion and can be quite fragile. Grasping the coronal aspect of a silver point, without any preparation to loosen it, risks fracturing the silver point. ${ }^{5}$

No standardized procedure for the successful removal of silver point in the canal exists, rather there are various techniques and devices that are established, and are used according to the situation. Braiding technique, involves the use of several Hedstrom files inserted along the length of the silver point and the files are twisted to grasp the silver point and then withdrawn as one unit. ${ }^{6}$ If the point is protruding into the pulp chamber then a sharp spoon excavator or curette can be used to loosen point from seat. A more efficient spoon excavator has been marketed by Stardent with a triangular notch cut out in the tip of the blade (Fig. 11). Specially designed pliers (Stieglitz pliers) can also be used to grasp and retrieve the partially exposed silver point. ${ }^{1}$

Ultrasonic instruments can also be used for silver point removal. However, if ultrasonic instruments are applied directly to the silver point, the portion in contact may be shredded, leaving a smaller segment to work with because elemental silver rapidly erodes during mechanical manipulation. ${ }^{2}$ If the silver point resists removal, ultrasonic vibrations can be applied indirectly. Placing a fine Hedstrom file down into the canal alongside the silver point, the file is then activated by ultrasonics (Fig. 12). It will loosen the obstruction and thus silver point can be retrieved. The clinician should always work at the lowest power setting that will efficiently and safely accomplish the clinical task.

A technique as devised by Rowe, uses cyanoacrylate glue (Permabond or Super Glue \#30) and hypodermic needles to retrieve silver point. ${ }^{1}$ According to it, when a silver point is not protruding into the pulp chamber then select a hypodermic needle that fits snugly over silver point. Remove the bevel of needle and cement it over the silver point using cyanoacrylate glue. After 5 minutes of setting time, the needle is grasped with hemostat and silver point worried from place (Fig. 13A). A variation of this method uses a larger gauge needle and a small Hedstrom file. ${ }^{1}$ The piece of blunted needle is placed over the butt end of the silver point. The file is then inserted down the inside of the needle and wedged tightly into the space between (Fig. 13B). Technique using orthodontic ligature wire and plastic tubing can also be used. In this, a groove is cut around the protruding butt end of the silver point with a half round or wheel bur. The ligature wire is then doubled over, and the 
two free ends are passed through the tubing to form a loop at the end. The groove in the silver point is then lassoed with the wire loop, which is cinched up tight with the plastic sleeve. Adding a drop of cyanoacrylate cement may improve the grip. ${ }^{1}$ The tubing is tightly grasped with pliers or a hemostat and the point is worked loose (Fig. 13C).

Silver points were designed to correspond to the last file size used in the preparation, to presumably fill the canal precisely in all dimensions. Because of the complexity of shape of the root canals, this is fallacious; it is impossible to predictably prepare canals to a uniform size and shape. Although the short term sealability success of silver points seemed comparable to that of gutta-percha, silver points are a poor long-term choice as routine obturating material.

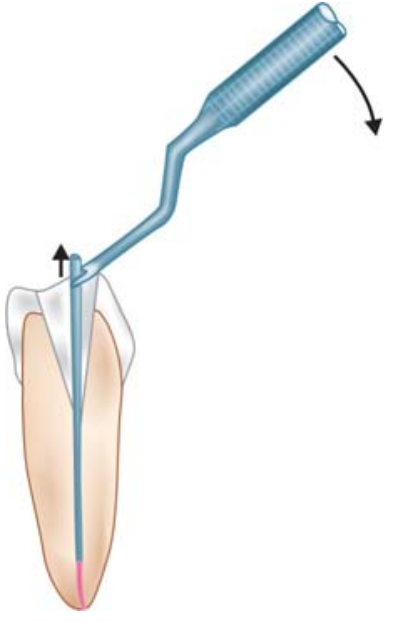

Fig. 11: Silver point removal technique using spoon excavator with a triangular notch cut (Courtesy: Ingle JI, Bakland LK, LK, Endodontics (5th ed). Endodontics (5th ed). Hamilton, Hamilton, London: BC Decker London: BC Decker Inc 2002; 648) Inc 2002;649)
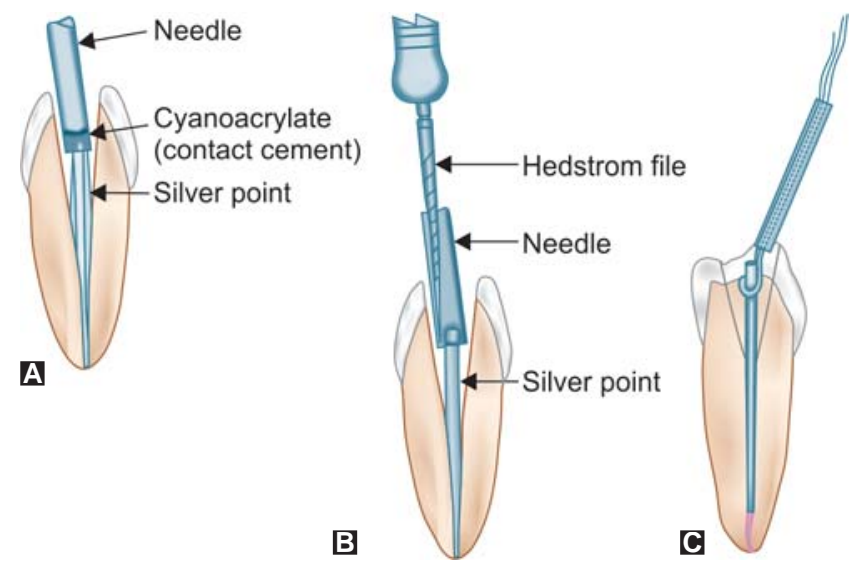

Figs 13A to C: Demonstrating different techniques of silver point removal, using $(A)$ cyanoacrylate glue and hypodermic needle, (B) larger gauze needle and $\mathrm{H}$-file, (C) orthodontic ligature wire and plastic tubing (Courtesy: Ingle JI, Bakland LK, Endodontics (5th ed). Hamilton, London: BC Decker Inc 2002;648)
Their major problems relate to nonadaptability and toxicity from corrosion. Also, because of their tight frictional fit and hardness, silver cones are difficult to remove totally or partially. Also, if silver cones are contacted with a bur, their seal may be broken. ${ }^{7}$ Hence, their use should be avoided when better alternatives such as gutta-percha and Resilon root filling techniques are available which do not have the above cited disadvantages.

\section{CONCLUSION}

Silver points do not adequately seal the apical foramen. These points corrode over time and allow high levels of leakage. Consequently, the use of silver points in root canal therapy exhibits a relatively high degree of failure, which often necessitates the retreatment of the affected tooth. Occasionally, if the silver points cannot be grasped, retrieval of the point is impossible which remarkably decrease the prognosis. Therefore, silver points are contraindicated as an obturating material in clinical practice.

\section{REFERENCES}

1. Ingle JI, Bakland LK. Endodontics (5th ed). Hamilton, London: BC Decker Inc 2002;645-49.

2. Cohen S, Hargreaves K. Pathways of the Pulp (9th ed). St Louis, Missouri: Elsevier Mosby 2006;522-24.

3. Nehme WB. Elimination of intracanal metallic obstructions by abrasion using an operational microscope and ultrasonics. J Endod 2001;27(5):365-67.

4. Ruddle CJ. Nonsurgical retreatment: Review. J Endod 2004;30(12):827-45

5. Ruddle CJ. Microendodontic nonsurgical retreatment: Silver point removal. Dent Today 1997 Feb;16(2):64-69.

6. Removal of separated files from root canals with a new file removal system: Case reports. J Endod 2006;32(8):789-97.

7. Walton RE, Torabinejad M. Principles and Practice Endodontics (3rd ed). Philadelphia: Elsevier Saunders 2002;240-67.

\section{ABOUT THE AUTHORS}

\section{Shikha Gulati (Corresponding Author)}

Postgraduate Student, Department of Conservative Dentistry and Endodontics, MGM Dental College and Hospital, Navi Mumbai Maharashtra, India, e-mail: vkshikha@gmail.com

\section{Sumanthini}

Professor, Department of Conservative Dentistry and Endodontics MGM Dental College and Hospital, Navi Mumbai, Maharashtra, India

\section{Vanitha Shenoy}

Professor and Head, Department of Conservative Dentistry and Endodontics, MGM Dental College and Hospital, Navi Mumbai Maharashtra, India 